\title{
EIGEN VALUE APPROACH TO TWO DIMENSIONAL PROBLEM IN GENERALIZED MAGNETO MICROPOLAR THERMOELASTIC MEDIUM WITH ROTATION EFFECT
}

\author{
R. SINGH \\ Department of Mathematics \\ S.G.A.D. Govt. College, Tarn Taran, Punjab, INDIA \\ E-mail:kalsi_ranjit@yahoo.com \\ V. KUMAR ${ }^{*}$ \\ Department of Mathematics \\ Lovely Professional University, Punjab, INDIA \\ E-mail: varun.kumar@hotmail.co.in
}

\begin{abstract}
In this study an eigen value approach has been employed to examine the mechanical force applied along with a transverse magnetic field in a two dimensional generalized magneto micropolar thermoelastic infinite space. Results have been obtained by treating rotational velocity to be invariant. Integral transforms have been applied to solve the system of partial differential equations. Components of displacement, normal stress, tangential couple stress, temperature distribution, electric field and magnetic field have been obtained in the transformed domain. Finally numerical inversion technique has been used to invert the result in the physical domain. Graphical analysis has been done to described the study.
\end{abstract}

Key words: magneto micropolar, thermoelasticity, eigen value, elasticity, integral transforms.

\section{Introduction}

The micropolar theory of elasticity was developed with the possibilities of its wide-ranging practical applications in diverse fields such as geophysics, optics and acoustics and so on. Contemporary engineering materials are usually made up of constituents possessing internal structures. Some of the material in this category are polycrystalline materials, materials with fibrous or coarse grain. Classical elasticity is inadequate to represent the behaviour of such materials. An analysis of these type of materials requires a special theory "Micropolar Elasticity" developed by Eringen [1] which deals with deformation of oriented particles. Basically a micropolar continuum is a collection of interconnected particles in the form of small rigid bodies which can undergo both translational and rotational motions. Classic examples of such materials are granular media and multimolecular bodies, whose microstructure act as an evident part in their macroscopic responses.

The current area of study namely: magneto micropolar thermoelasticity is an extension of this theory. This theory deals with the effects of the magnetic field on the elastic deformation produced by uneven heating throughout the body which may or may not be subjected to mechanical forces. In this case, in addition to elastic and electro-magnetic fields, thermal field is also present. Each of these fields contributes to the total deformation of the body and interacts with each other. Maxwell's equations still govern the electro-magnetic field while the elastic field is determined by the modified Hooke's law and the thermal field by Fourier's law of heat conduction in its modified form. Due to superposition of the electromagnetic field

\footnotetext{
* To whom correspondence should be addressed
} 
on the elastic field, the elastic-stress relation gets modified with the introduction of Lorentz's force as body force and in turn the elastic field influences the electro-magnetic field by modifying Ohm's law.

Basic equations of magneto micro thermoelasticity were obtained by Kaliski [2]. Also, the wave type of the above equations were studied by Kaliski and Nowacki [3]. Paria [4], Knopoff [5], Banos [6], Chadwick [7], and Purushothama [8] contributed to magneto elasticity theories. Nowacki [9] studied a simplified two dimensional problem of magneto-micropolar elasticity. Ezzat and Youssef [10] investigated the problem of micropolar thermal elasticity in perfectly conducting media. Bakasi et al. [11] studied magneto thermal elastic problems with thermal relaxations and heat sources in a three dimensional infinite rotating elastic media. A problem of generalized magneto thermoelasticity in a conducting medium with variable material properties was also studied by Youssef [12]. The effect of rotation was analyzed by Kumar and Rupender [13] by using a two dimensional model in an electromagnetic micropolar generalized thermoelastic medium in the presence of a transverse magnetic field subjected to a mechanical force or thermal source and observed that the application of a thermal source is more significant than the mechanical force. Ezzat and Bary [14] compared the one-temperature theory with the two temperature theory in a generalized magneto thermoelastic medium in a perfectly conducting medium using the state space approach subjected to a thermal shock and traction-free surface and found that the two-temperature generalized theory describes the behavior of the particles of an elastic body more accurately than the one-temperature theory. Ezzat and Awad [15] introduced the modified Ohm's law, including the temperature gradient and charge density effects, and the generalized Fourier's law including current density effect to the equations of the linear theory of micropolar generalized magneto thermoelasticity. A normal mode analysis is used to obtain the solution. He and Cao [16] used the generalized thermoelastic theory with thermal relaxation in the context of L-S theory to investigate the magneto thermoelastic problem of a thin slim strip placed in a magnetic field and subjected to a moving plane of heat source and found that the magnetic field significantly influences the variations of non-dimensional displacement and stress but has no effect on the nondimensional temperature. Singh and Kumar [17] studied the interaction of the electromagnetic field with the elastic field in the presence of temperature by applying the mechanical force and thermal source by using modified Fourier and Ohm's law.

Increasing attention is devoted to the interaction between magnetic fields and strain in a micropolar thermoelastic solid due to many applications in the fields of geophysics, plasma physics and related areas. The deformation at any point of the medium is useful to analyze the deformation field around mining tremors and drilling into the crust of the earth. It may also find application in various engineering problems, crystal physics and solid-earth geophysics. The present study can be regarded as a better representation of the elastic model for studying the earth's planetary motion as it involves rotational velocity in addition to its thermal and electromagnetic field. The scope of the present study is to examine the interaction in the magneto micropolar thermoelastic material due to a mechanical source.

\section{Basic equations}

Following Baksi et al. [11], the linear equations of electrodynamics of a slowly moving medium for a homogenous and perfectly conducting elastic solid in the simplified form along with field equations of motion and constitutive relations in the theory of micropolar generalized thermoelasticity, taking into account the Lorentz force are given by Eqs (2.1)-(2.9)

$$
\begin{aligned}
& \operatorname{Curl} \boldsymbol{h}=\mathrm{J}+\epsilon_{0} \frac{\partial \boldsymbol{E}}{\partial t}, \\
& \operatorname{Curl} \boldsymbol{E}=-\frac{\partial \boldsymbol{h}}{\partial t}, \\
& \boldsymbol{E}=-\mu_{0}\left(\frac{\partial \boldsymbol{u}}{\partial t} \times \boldsymbol{H}_{o}\right),
\end{aligned}
$$




$$
\begin{aligned}
& \operatorname{div} \boldsymbol{h}=0, \\
& (\lambda+2 \mu+K) \nabla(\nabla \cdot \boldsymbol{u})-(\mu+K) \nabla \times(\nabla \times \boldsymbol{u})+K(\nabla \times \phi)+\boldsymbol{F}-\mathrm{v}\left(1+\tau_{1} \frac{\partial}{\partial t}\right) \nabla T= \\
& =\rho\left[\frac{\partial^{2} \boldsymbol{u}}{\partial t^{2}}+\boldsymbol{\Omega} \times(\boldsymbol{\Omega} \times \boldsymbol{u})+2 \boldsymbol{\Omega} \times \frac{\partial \boldsymbol{u}}{\partial t}\right], \\
& (\alpha+\beta+\gamma) \nabla(\nabla \cdot \phi)-\gamma \nabla \times(\nabla \times \phi)+K(\nabla \times \boldsymbol{u})-2 K \phi=\rho j\left[\frac{\partial^{2} \phi}{\partial t^{2}}+\boldsymbol{\Omega} \times \frac{\partial \phi}{\partial t}\right], \\
& K^{*} \nabla^{2} T=\rho c^{*}\left(\frac{\partial}{\partial t}+\tau_{o} \frac{\partial^{2}}{\partial t^{2}}\right) T+v T_{o}\left(\frac{\partial}{\partial t}+\tau_{o} \eta_{o} \frac{\partial^{2}}{\partial t^{2}}\right)(\nabla \cdot \boldsymbol{u}), \\
& \sigma_{i j}=\lambda u_{k, k} \delta_{i j}+\mu\left(u_{i, j}+u_{j, i}\right)+K\left(u_{j, i}-\epsilon_{i j k} \phi_{k}\right)-v\left(1+\tau_{l} \frac{\partial}{\partial t}\right) T \delta_{i j}, \\
& m_{i j}=\alpha \phi_{k, k} \delta_{i j}+\beta \phi_{i, j}+\gamma \phi_{j, i}
\end{aligned}
$$

where

$$
v=(3 \lambda+2 \mu+K) \alpha_{t} \quad \text { and } \quad \boldsymbol{F}=\mu_{0}\left(\boldsymbol{J} \times \boldsymbol{H}_{o}\right)
$$

$\boldsymbol{H}_{o}$ is the external applied magnetic field intensity vector, $\boldsymbol{h}$ the induced magnetic field vector, $\boldsymbol{E}$ the induced electric field vector, $\boldsymbol{J}$ the current density vector, $\boldsymbol{u}$ the displacement vector, $\boldsymbol{\mu}_{0}$ and $\in_{0}$ the magnetic and electric permeabilities, respectively, and $\delta_{i j}$ - the Kroneker delta.

\section{Formulation and solution of the problem}

We consider a homogenous, isotropic, perfectly conducting micropolar generalized thermoelastic medium, permeated by an initial magnetic field $\boldsymbol{H}_{o}$ acting along the $x_{2}$-axis.

For a two dimensional problem we take the displacement vector $\boldsymbol{u}$, rotation vector $\boldsymbol{\Omega}$ and microrotation vector $\phi$ as (by assuming $\boldsymbol{\Omega}$ to be invariant)

$$
\begin{aligned}
& \boldsymbol{u}=\left(u_{1}, 0, u_{3}\right), \quad \phi=\left(0, \phi_{2}, 0\right), \quad \boldsymbol{\Omega}=\left(0, \Omega_{2}, 0\right), \quad \boldsymbol{E}=\left(E_{1}, 0, E_{3}\right), \\
& \boldsymbol{h}=(0, h, 0), \quad \boldsymbol{H}_{0}=\left(0, H_{02}, 0\right) .
\end{aligned}
$$

Using expressions mentioned in Eq.(3.1) in Eqs (2.1)-(2.4), (2.8)-(2.9) we get

$$
\begin{aligned}
& (\lambda+2 \mu+K) \frac{\partial^{2} u_{1}}{\partial x_{1}^{2}}+(\lambda+\mu) \frac{\partial^{2} u_{3}}{\partial x_{1} \partial x_{3}}+(\mu+K) \frac{\partial^{2} u_{1}}{\partial x_{3}^{2}}-K \frac{\partial \phi_{2}}{\partial x_{3}}+ \\
& -\mu_{0} H_{02} J_{3}-v\left(1+\tau_{1} \frac{\partial}{\partial t}\right) \frac{\partial T}{\partial x_{1}}=\rho\left[\frac{\partial^{2} u_{1}}{\partial t^{2}}-3 \Omega_{2}^{2} u_{1}\right]
\end{aligned}
$$




$$
\begin{aligned}
& (\lambda+\mu) \frac{\partial^{2} u_{3}}{\partial x_{1} \partial x_{3}}+(\lambda+2 \mu+K) \frac{\partial^{2} u_{3}}{\partial x_{3}^{2}}+(\mu+K) \frac{\partial^{2} u_{3}}{\partial x_{1}^{2}}+K \frac{\partial \phi_{2}}{\partial x_{3}}+ \\
& +\mu_{0} H_{0} J_{1}-v\left(1+\tau_{1} \frac{\partial}{\partial t}\right) \frac{\partial T}{\partial x_{3}}=\rho\left[\frac{\partial^{2} u_{3}}{\partial t^{2}}-3 \Omega_{2}^{2} u_{3}\right] \\
& -\gamma\left(\frac{\partial^{2} \phi_{2}}{\partial x_{1}^{2}}+\frac{\partial^{2} \phi_{2}}{\partial x_{3}^{2}}\right)+K\left(\frac{\partial u_{1}}{\partial x_{3}}-\frac{\partial u_{3}}{\partial x_{1}}\right)-2 K \phi_{2}=\rho j \frac{\partial^{2} \phi_{2}}{\partial t^{2}} \\
& K^{*} \nabla^{2} T=\rho c^{*}\left(\frac{\partial}{\partial t}+\tau_{o} \frac{\partial^{2}}{\partial t^{2}}\right) T+v T_{o}\left(\frac{\partial}{\partial t}+\tau_{o} \eta_{o} \frac{\partial^{2}}{\partial t^{2}}\right)\left(\frac{\partial u_{1}}{\partial x_{1}}+\frac{\partial u_{3}}{\partial x_{3}}\right) \\
& \sigma_{31}=\mu \frac{\partial u_{3}}{\partial x_{1}}+(\mu+K) \frac{\partial u_{1}}{\partial x_{3}}+K \phi_{2} \\
& \sigma_{33}=\lambda \frac{\partial u_{1}}{\partial x_{1}}+(\lambda+2 \mu+K) \frac{\partial u_{3}}{\partial x_{3}}-v\left(1+\tau_{1} \frac{\partial}{\partial t}\right) T \\
& E_{32}=\gamma \frac{\partial \phi_{2}}{\partial x_{3}}, \\
& E_{1}=\mu_{0} H_{02} \frac{\partial u_{3}}{\partial t} \\
& h=\mu_{02} H_{02} \frac{\partial u_{1}}{\partial t}, \\
& m_{1}
\end{aligned}
$$

We define the dimensionless quantities as

$$
\begin{aligned}
& x_{i}^{*}=\frac{\bar{\omega}}{c_{1}} x_{i}, \quad u_{i}^{*}=\frac{\rho c_{1} \bar{\omega}}{v T_{0}} u_{i}, \quad t^{*}=\bar{\omega} t, \quad \tau_{0}{ }^{*}=\bar{\omega} \tau_{0}, \quad \tau_{1}^{*}=\bar{\omega} \tau_{1}, \\
& J_{i}^{*}=\frac{\eta_{0}}{\sigma_{0}{ }^{2} \mu_{0}{ }^{2} H_{02} c_{0}} J_{i}, \quad h^{*}=\frac{\eta_{0}}{\sigma_{0} \mu_{0} H_{02}} h, \quad \sigma_{i j}{ }^{*}=\frac{1}{v T_{0}} \sigma_{i j}, \quad m_{i j}{ }^{*}=\frac{\bar{\omega}}{c_{1} v T_{0}} m_{i j}, \\
& E_{i}^{*}=\frac{E_{i}}{\mu_{0} H_{02} c_{1}}, \quad \Omega_{2}^{*}=\frac{\Omega_{2}}{\bar{\omega}}, \quad T^{*}=\frac{v T}{\rho c_{0}{ }^{2}}, \quad \phi_{2}^{*}=\frac{\rho c_{1}^{2} \bar{\omega}}{v T_{0}} \phi_{2}, \quad \text { for } \quad i=1.3
\end{aligned}
$$

where 


$$
c_{1}^{2}=\frac{\lambda+2 \mu+\kappa}{\rho} \quad \text { and } \quad \bar{\omega}=\frac{\rho c^{*} c_{1}^{2}}{K} .
$$

Using the dimensionless quantities as defined in Eq.(3.12), the system of Eqs (3.2)-(3.11) after suppressing the asterisks can be rewritten as

$$
\begin{aligned}
& \left(\alpha_{1}+\alpha_{5}\right) \frac{\partial^{2} u_{1}}{\partial x_{1}^{2}}+\left(\alpha_{2}+\alpha_{5}\right) \frac{\partial^{2} u_{3}}{\partial x_{1} \partial x_{3}}+\alpha_{3} \frac{\partial^{2} u_{1}}{\partial x_{3}^{2}}-\alpha_{4} \frac{\partial \phi_{2}}{\partial x_{3}}+ \\
& -\alpha_{7}\left(1+\tau_{1} \bar{\omega} \frac{\partial}{\partial t}\right) \frac{\partial T}{\partial x_{1}}=\left(\alpha_{6}+\alpha_{7}\right) \frac{\partial^{2} u_{1}}{\partial t^{2}}-\alpha_{9} u_{1}, \\
& \alpha_{3} \frac{\partial^{2} u_{3}}{\partial x_{1}^{2}}+\left(\alpha_{2}+\alpha_{5}\right) \frac{\partial^{2} u_{1}}{\partial x_{1} \partial x_{3}}+\left(\alpha_{1}+\alpha_{5}\right) \frac{\partial^{2} u_{3}}{\partial x_{3}^{2}}+\alpha_{4} \frac{\partial \phi_{2}}{\partial x_{1}}+ \\
& -\alpha_{7}\left(1+\tau_{1} \bar{\omega} \frac{\partial}{\partial t}\right) \frac{\partial T}{\partial x_{3}}=\left(\alpha_{6}+\alpha_{7}\right) \frac{\partial^{2} u_{3}}{\partial t^{2}}-\alpha_{9} u_{3}, \\
& -\alpha_{11}\left(\frac{\partial^{2} \phi_{2}}{\partial x_{1}^{2}}+\frac{\partial^{2} \phi_{2}}{\partial x_{3}^{2}}\right)+\alpha_{12}\left(\frac{\partial u_{1}}{\partial x_{3}}-\frac{\partial u_{3}}{\partial x_{1}}\right)-\alpha_{13} \phi_{2}=\alpha_{14} \frac{\partial^{2} \phi_{2}}{\partial t^{2}} \\
& \alpha_{15} \nabla^{2} T=\alpha_{16}\left(\frac{\partial}{\partial t}+\tau_{o} \bar{\omega} \frac{\partial^{2}}{\partial t^{2}}\right) T+\alpha_{17}\left(\frac{\partial}{\partial t}+\tau_{o} \eta_{o} \bar{\omega} \frac{\partial^{2}}{\partial t^{2}}\right)\left(\frac{\partial u_{1}}{\partial x_{1}}+\frac{\partial u_{3}}{\partial x_{3}}\right) \\
& E_{1}=\alpha_{24} \frac{\partial u_{3}}{\partial t}, \\
& E_{31}=-\alpha_{24} \frac{\partial u_{1}}{\partial t}, \\
& m_{32}=\alpha_{23} \frac{\partial \phi_{3}}{\partial x_{1}}+\alpha_{21} \frac{\partial u_{1}}{\partial x_{3}}+\alpha_{22} \phi_{2}, \\
& \sigma_{33}=\alpha_{18} \frac{\partial u_{1}}{\partial x_{1}}+\alpha_{19} \frac{\partial u_{3}}{\partial x_{3}}-\alpha_{20} T \\
& \left.\frac{\partial u_{1}}{\partial x_{1}}+\frac{\partial u_{3}}{\partial x_{3}}\right)
\end{aligned}
$$

where 


$$
\begin{aligned}
& \alpha_{1}=\frac{(\lambda+2 \mu+K)}{\rho c_{1}^{3}} v T_{0} \bar{\omega}, \quad \alpha_{2}=\frac{(\lambda+\mu)}{\rho c_{1}^{3}} v T_{0} \bar{\omega}, \quad \alpha_{3}=\frac{(\mu+K)}{\rho c_{1}^{3}} v T_{0} \bar{\omega}, \\
& \alpha_{4}=\frac{K v T_{0} \bar{\omega}}{\rho c_{1}^{3}}, \quad \alpha_{5}=\frac{\mu_{0} H_{02}^{2} v T_{0} \bar{\omega}}{\rho c_{1}^{3}}, \quad \alpha_{6}=\frac{\varepsilon_{0} \mu_{0}^{2} H_{02}^{2} v T_{0} \bar{\omega}}{\rho c_{1}}, \quad \alpha_{7}=\frac{v T_{0} \bar{\omega}}{c_{1}}, \\
& \alpha_{8}=\frac{v T_{0} \bar{\omega}}{c_{1}}, \quad \alpha_{9}=\frac{3 \Omega_{2}^{2} K v T_{0} \bar{\omega}}{\rho c_{1}^{3}}, \quad \alpha_{11}=\frac{\gamma v T_{0} \bar{\omega}^{2}}{\rho c_{1}^{4}}, \quad \alpha_{12}=\frac{K v T_{0}}{\rho c_{1}^{2}}, \\
& \alpha_{13}=\frac{2 K v T_{0}}{\rho c_{1}^{2}}, \quad \alpha_{14}=\frac{j v T_{0} \bar{\omega}^{2}}{c_{1}^{2}}, \quad \alpha_{15}=\frac{K^{*} T_{0} \bar{\omega}^{2}}{c_{1}^{2}}, \quad \alpha_{16}=\rho \bar{\omega} T_{0}, \quad \alpha_{17}=\frac{v^{2} T_{0}^{2} \bar{\omega}}{\rho c_{1}^{2}}, \\
& \alpha_{18}=\frac{\lambda}{\rho c_{1}^{2}}, \quad \alpha_{19}=\frac{(\lambda+2 \mu+K)}{\rho c_{1}^{2}}, \quad \alpha_{20}=\frac{\mu}{\rho c_{1}^{2}}, \quad \alpha_{21}=\frac{\mu+K}{\rho c_{1}^{2}}, \quad \alpha_{22}=\frac{K}{\rho c_{1}^{2}}, \\
& \alpha_{23}=\frac{\gamma \bar{\omega}^{2}}{\rho c_{1}^{4}}, \quad \alpha_{24}=\frac{v T_{0}}{\rho c_{1}^{2}}, \quad \alpha_{25}=\frac{v T_{0}}{\rho c_{1}^{2} \bar{\omega}} .
\end{aligned}
$$

We take the Laplace and Fourier transform as

$$
\begin{aligned}
& L\left\{f\left(x_{1}, x_{3}, t\right)\right\}=\int_{0}^{\infty} e^{-s t} f\left(x_{1}, x_{3}, t\right) d t=\bar{f}\left(x_{1,} x_{3}, s\right) \\
& F\left\{\bar{f}\left(x_{1}, x_{3}, s\right)\right\}=\int_{-\infty}^{\infty} e^{-i x_{1}, \xi} f\left(x_{1}, x_{3}, t\right) d t=\tilde{f}\left(\xi, x_{3}, t\right)
\end{aligned}
$$

After applying the transformation as defined in Eqs (3.24)-(3.25) on Eqs (3.13)-(3.16), we get

$$
\begin{aligned}
& D^{2} \tilde{u}_{1}=\frac{1}{\alpha_{3}}\left[\left\{\left(\alpha_{1}+\alpha_{5}\right) \xi^{2}+\left(\alpha_{6}+\alpha_{8}\right) s^{2}-\alpha_{9}\right\} \tilde{u}_{1}-i \xi\left(\alpha_{2}+\alpha_{5}\right) D \tilde{u}_{3}+\right. \\
& \left.+\alpha_{4} D \tilde{\phi}_{2}-i \xi \alpha_{7}\left(1+\bar{\omega} \tau_{1}\right) \tilde{T}\right], \\
& D^{2} \tilde{u}_{3}=\frac{1}{\alpha_{1}+\alpha_{5}}\left[-i \xi\left(\alpha_{2}+\alpha_{5}\right) D \tilde{u}_{1}+\left\{\alpha_{3} \xi^{2}+\left(\alpha_{6}+\alpha_{8}\right) s^{2}-\alpha_{9}\right\} \tilde{u}_{3}+\right. \\
& \left.-i \xi \alpha_{4} \tilde{\phi}_{2}+\alpha_{7}\left(1+\bar{\omega} \tau_{1} s\right) D \tilde{T}\right], \\
& D^{2} \tilde{\phi}_{2}=\frac{1}{\alpha_{11}}\left[\alpha_{12}\left(D \tilde{u}_{1}-i \xi \tilde{u}_{3}\right)+\left(\alpha_{11} \xi^{2}-\alpha_{13}-\alpha_{14} s^{2}\right) \tilde{\phi}_{2}\right],
\end{aligned}
$$




$$
\begin{aligned}
& D^{2} \tilde{T}=\frac{1}{\alpha_{15}}\left[i \xi \alpha_{17}\left(s+\tau_{0} \eta_{0} \bar{\omega} s^{2}\right) \tilde{u}_{1}+\alpha_{17}\left(s+\tau_{0} \eta_{0} \bar{\omega} s^{2}\right) D \tilde{u}_{3}+\left(\alpha_{15} \xi^{2}+s+\bar{\omega} \tau_{0} s^{2}\right) \tilde{T}\right] \\
& \tilde{\sigma}_{31}=i f_{52} \tilde{u}_{3}+\alpha_{21} D \tilde{u}_{1}+\alpha_{22} \tilde{\phi}_{2} \\
& \tilde{\sigma}_{33}=i f_{51} \tilde{u}_{1}+\alpha_{19} D \tilde{u}_{3}-\alpha_{20} \tilde{T} \\
& \tilde{m}_{32}=\alpha_{23} D \tilde{\phi}_{2} \\
& \tilde{E}_{1}=f_{53} \tilde{u}_{3} \\
& \tilde{E}_{3}=-f_{53} \tilde{u}_{1} \\
& \tilde{h}=-i f_{54} \tilde{u}_{1}-\alpha_{25} D \tilde{u}_{3}
\end{aligned}
$$

where $\quad D=\frac{d}{d z}$.

Equations (3.26)-(3.29) can be written in a matrix form as

$$
D W\left(\xi, x_{3}, s\right)=A W\left(\xi, x_{3}, s\right)
$$

where

$$
\begin{aligned}
& W=\left[\begin{array}{c}
U \\
D U
\end{array}\right], \quad U=\left[\begin{array}{llll}
\tilde{u}_{1} & \tilde{u}_{3} & \tilde{\phi}_{2} & \tilde{T}
\end{array}\right]^{\prime}, \\
& A=\left[\begin{array}{cc}
O & I \\
A_{2} & A_{1}
\end{array}\right], A_{1}=\left[\begin{array}{cccc}
0 & -i g_{12} & g_{13} & 0 \\
i g_{21} & 0 & 0 & g_{24} \\
g 31 & 0 & 0 & 0 \\
0 & g_{42} & 0 & 0
\end{array}\right], A_{2}=\left[\begin{array}{cccc}
f_{11} & 0 & 0 & -i f_{14} \\
0 & f_{22} & i f_{23} & 0 \\
0 & -i f_{32} & f_{33} & 0 \\
i f_{41} & 0 & 0 & f_{44}
\end{array}\right],
\end{aligned}
$$

$I$ is identity matrix of order 4, $O$ is null matrix of order of 4 and []$^{\prime}$ is the transpose of matrix.

Where

$$
\begin{aligned}
& f_{11}=\frac{1}{\alpha_{3}}\left[\left(\alpha_{1}+\alpha_{5}\right) \xi^{2}+\left(\alpha_{6}+\alpha_{8}\right) s^{2}-\alpha_{9}\right], \quad f_{14}=\frac{\xi \alpha_{7}}{\alpha_{3}}\left(1+\tilde{\omega} \tau_{1} s\right), \\
& f_{22}=\frac{\alpha_{3} \xi^{2}+\alpha_{6} s^{2}+\alpha_{8} s^{2}-\alpha_{9}}{\alpha_{1}+\alpha_{5}}, \quad f_{23}=-\frac{\alpha_{4} \xi}{\alpha_{1}+\alpha_{5}}, \quad f_{32}=\frac{\xi \alpha_{12}}{\alpha_{11}}, \\
& f_{33}=\frac{\alpha_{11} \xi^{2}-\alpha_{14} s^{2}-\alpha_{13}}{\alpha_{11}}, \quad f_{41}=-\frac{\xi \alpha_{17}}{\alpha_{15}}\left(s+\tau_{0} \eta_{0} \bar{\omega} s^{2}\right),
\end{aligned}
$$




$$
\begin{aligned}
& f_{44}=\frac{1}{\alpha_{15}}\left(\alpha_{15} \xi^{2}+s+\tau_{0} \bar{\omega} s^{2}\right), \quad g_{12}=-\frac{\xi\left(\alpha_{2}+\alpha_{5}\right)}{\alpha_{3}}, \quad g_{13}=\frac{\alpha_{4}}{\alpha_{3}}, \\
& g_{21}=-\frac{\xi\left(\alpha_{5}+\alpha_{2}\right)}{\alpha_{1}+\alpha_{5}}, \quad g_{24}=\frac{\alpha_{7}}{\alpha_{1}+\alpha_{5}}\left(1+\tau_{1} \bar{\omega} s\right), \quad g_{31}=\frac{\alpha_{12}}{\alpha_{11}}, g_{42}=\frac{\alpha_{17}}{\alpha_{15}}\left(s+\tau_{0} \eta_{0} \bar{\omega} s^{2}\right) .
\end{aligned}
$$

To solve the above equation, we take $W\left(\xi, x_{3}, s\right)=X(\xi, s) e^{q x_{3}}$, for some parameter $q$.

Using this value in Eq.(3.36), we get

$$
A W\left(\xi, x_{3}, s\right)=q W\left(\xi, x_{3}, s\right)
$$

which leads to the eigen value problem.

A characteristic equation corresponding to the matrix $A$ is given as

$$
|A-q I|=0
$$

which on expansion gives

$$
q^{8}-\lambda_{1} q^{6}+\lambda_{2} q^{4}-\lambda_{3} q^{2}+\lambda_{4}=0
$$

where

$$
\begin{aligned}
& \lambda_{1}=g_{24} g_{4}+f_{11}+f_{44}+f_{22}+f_{33}-g_{12} g_{21}+g_{13} g_{31}, \\
& \lambda_{2}=-f_{41} g_{12} g_{24}+f_{14} f_{41}+g_{13} g_{24} g_{31} g_{42}+f_{33} g_{24} g_{42}+f_{11} g_{42}-f_{14} f_{21} g_{42}+f_{11} f_{44}+ \\
& +f_{11 f_{22}}+f_{11} f_{33}+f_{22} f_{44}+f_{33} f_{44}+f_{22} f_{33}+f_{23} f_{32}-f_{44} g_{12} g_{21}-f_{33} g_{12} g_{21}+ \\
& +f_{23} g_{12} g_{31}+f_{44} g_{13} g_{31}+f_{32} g_{13} g_{21}+f_{22} g_{13} g_{31}, \\
& \lambda_{3}=-f_{32} f_{41} g_{13} g_{24}-f_{33} f_{41} g_{12} g_{24}+f_{14} f_{33} f_{41}+f_{14} f_{22} f_{41}+f_{14} f_{23} g_{31} g_{42}+ \\
& +f_{11} f_{33} g_{42}-f_{14} f_{21} f_{33} g_{42}+f_{22} f_{11} f_{44}+f_{11} f_{33} f_{44}+f_{11} f_{22} f_{33}+ \\
& +f_{22} f_{33} f_{44}+f_{23} f_{32} f_{44}-f_{33} f_{44} g_{12} g_{21}+f_{23} f_{44} g_{12} g_{31}+f_{32} f_{44} g_{13} g_{21}+f_{22} f_{44} g_{13} g_{31}, \\
& \lambda_{4}=f_{14} f_{23} f_{32} f_{41}+f_{14} f_{33} f_{41} f_{22}+f_{11} f_{22} f_{33} f_{44}+f_{11} f_{23} f_{32} f_{44} .
\end{aligned}
$$

The eigen values of matrix $A$ are the characteristic roots of Eq.(3.42). The eigen vectors $X(\xi, s)$ corresponding to eigen value $q_{p}$ can be determined by solving the homogenous equations

$$
[A-q I] X(\xi, s)=0,
$$

which gives 


$$
X_{p}(\xi, s)=\left[\begin{array}{c}
X_{p 1} \\
X_{p 2}
\end{array}\right], \quad X_{p 1}=\left[\begin{array}{c}
a_{p} q_{p} \\
b_{p} \\
c_{p} q_{p} \\
d_{p}
\end{array}\right], \quad X_{p 2}=q_{p} X_{p 1} \quad \text { for } \quad q=q_{p}, \quad p=1,2,3,4
$$

and $\quad X_{j}(\xi, s)=\left[\begin{array}{c}X_{j 1} \\ X_{j 2}\end{array}\right], X_{j 1}=\left[\begin{array}{c}-a_{p} q_{p} \\ b_{p} \\ -c_{p} q_{p} \\ d_{p}\end{array}\right], X_{p 2}=q_{p} X_{p 1}$, for $j=p+4, \quad q=-q_{p}, \quad p=1,2,3,4$

where

$$
\begin{aligned}
& a_{p}=\left[\left\{-g_{12}\left(f_{44}-q_{p}^{2}\right)+i f_{44} g_{42}\right\}\left(f_{33}-q_{p}^{2}\right)-g_{13} g_{31} q_{p}^{2}\left(f_{44}-q_{p}^{2}\right)\right], \\
& b_{p}=\left[\left\{\left(f_{11}-q_{s}^{2}\right)\left(f_{44}-q_{p}^{2}\right)+i f_{14} f_{41}\right\}\left(f_{23}-q_{p}^{2}\right)-g_{13} g_{31} q_{p}^{2}\left(f_{44}-q_{p}^{2}\right)\right], \\
& c_{p}=\left[\left\{\left(f_{11}-q_{s}^{2}\right)\left(f_{44}-q_{p}^{2}\right)+f_{14} f_{41}\right\} f_{32}+g_{31} q_{p}^{2}\left\{-g_{12}\left(f_{44}-q_{p}^{2}\right)+i f_{14} g_{42}\right\}\right], \\
& d_{p}=-\frac{i\left(f_{41} a_{p}+g_{42} b_{p}\right)}{f_{44}-q_{p}^{2}} .
\end{aligned}
$$

Thus a solution of Eq.(3.40) becomes

$$
W(\xi, s)=\sum_{p=1}^{4}\left[B_{p} X_{p}(\xi, s) e^{q_{p} x_{3}}+B_{p+4} X_{p+4}(\xi, s) e^{-q_{p} x_{3}}\right]
$$

where $B_{i}^{\prime} s$ are eight arbitrary constants.

Now after using Eqs (3.26)-(3.35), (3.37) and (3.47), we obtain values of $\tilde{u}_{1}, \tilde{u}_{3}, \tilde{\phi}_{2}, \tilde{T}, \tilde{\sigma}_{31}$, $\tilde{\sigma}_{33}, \tilde{m}_{32}, \tilde{E}_{1}, \tilde{E}_{3}$ and $\tilde{h}$ as

$$
\begin{aligned}
& \tilde{u}_{1}=\sum_{p=1}^{4}\left[a_{p} q_{p} B_{p} e^{q_{p} x_{3}}-a_{p} q_{p} B_{p+4} e^{-q_{p} x_{3}}\right], \\
& \tilde{u}_{3}=\sum_{p=1}^{4}\left[b_{p} B_{p} e^{q_{p} x_{3}}+b_{p} B_{p+4} e^{-q_{p} x_{3}}\right] \\
& \tilde{\phi}_{2}=-\sum_{p=1}^{4}\left[c_{p} B_{p} e^{q_{p} x_{3}}+c_{p} B_{p+4} e^{-q_{p} x_{3}}\right]
\end{aligned}
$$




$$
\begin{aligned}
& \tilde{T}=\sum_{p=1}^{4}\left[d_{p} B_{p} e^{q_{p} x_{3}}+d_{p} B_{p+4} e^{-q_{p} x_{3}}\right], \\
& \tilde{\sigma}_{33}=\sum_{p=1}^{4}\left[\left(i a_{p} q_{p} f_{51}+\alpha_{19} b_{p} q_{p}-\alpha_{20} d_{p}\right) B_{p} e^{q_{p} x_{3}}+\right. \\
& \left.+\left(-i f_{51} a_{p} q_{p}-\alpha_{19} b_{p} q_{p}-\alpha_{20} d_{p}\right) B_{p+4} e^{-q_{p} x_{3}}\right], \\
& \tilde{\sigma}_{31}=\sum_{p=1}^{4}\left[\left(i f_{52} b_{p}+\alpha_{21} a_{p} q_{p}^{2}-\alpha_{22} c_{p}\right) B_{p} e^{q_{p} x_{3}}+\right. \\
& \left.+\left(i f_{52} b_{p}+\alpha_{21} a_{p} q_{p}^{2}-\alpha_{22} c_{p}\right) B_{p+4} e^{-q_{p} x_{3}}\right], \\
& \tilde{m}_{32}=-\alpha_{23} \sum_{p=1}^{4}\left[b_{p} c_{p} q_{p} B_{p} e^{q_{p} x_{3}}-c_{p} q_{p} B_{p+4} e^{-q_{p} x_{3}}\right], \\
& \left.+\left(i f_{54} a_{p} q_{p}+\alpha_{25} b_{p}-q_{p}\right) B_{p+4} e^{-q_{p} x_{3}}\right] . \\
& \tilde{E}_{1}=f_{53} \sum_{p=1}^{4}\left[b_{p} B_{p} e^{q_{p} x_{3}}+b_{p} B_{p+4} e^{-q_{p} x_{3}}\right], \\
& \tilde{h}=-i f_{54} \sum_{p=1}^{4}\left[\left(-i a_{p} q_{p} f_{54}+\alpha_{25} b_{p} q_{p}\right) B_{p} e^{q_{p} x_{3}}+\right. \\
& \tilde{E}_{33} \sum_{p=1}^{4}\left[a_{p} q_{p} B_{p} e^{q_{p} x_{3}}-a_{p} q_{p} B_{p+4} e^{-q_{p} x_{3}}\right],
\end{aligned}
$$

\section{Boundary conditions}

We consider an infinite micropolar elastic space in which a concentrated force $F=-P_{0} \delta\left(x_{1}\right) \delta(t)$ where $P_{0}$ is the magnitude of the force, acting in the direction of the $x_{3}$-axis at the origin. The boundary conditions for the present problem on the plane $x_{3}=0$ are

$$
\begin{aligned}
& u_{1}\left(x_{1}, 0^{+}, t\right)-u_{1}\left(x_{1}, 0^{-}, t\right)=0, \\
& u_{3}\left(x_{1}, 0^{+}, t\right)-u_{3}\left(x_{1}, 0^{-}, t\right)=0, \\
& \phi_{2}\left(x_{1}, 0^{+}, t\right)-\phi_{2}\left(x_{1}, 0^{-}, t\right)=0,
\end{aligned}
$$




$$
\begin{aligned}
& T\left(x_{1}, 0^{+}, t\right)-T\left(x_{1}, 0^{-}, t\right)=0, \\
& \frac{\partial T}{\partial x_{3}}\left(x_{1}, 0^{+}, t\right)-\frac{\partial T}{\partial x_{3}}\left(x_{1}, 0^{-}, t\right)=0, \\
& \sigma_{31}\left(x_{1}, 0^{+}, t\right)-\sigma_{31}\left(x_{1}, 0^{-}, t\right)=0, \\
& \sigma_{33}\left(x_{1}, 0^{+}, t\right)-\sigma_{33}\left(x_{1}, 0^{-}, t\right)=-P_{0} \delta\left(x_{1}\right) \delta(t), \\
& m_{32}\left(x_{1}, 0^{+}, t\right)-m_{32}\left(x_{1}, 0^{-}, t\right)=0 .
\end{aligned}
$$

After solving Eqs (4.1)-(4.8), we get

$$
\begin{aligned}
& B_{1}=B_{5}=\frac{P_{0} c_{4}}{l_{11} q_{1}}\left[\frac{l_{12} l_{23}-l_{13} l_{22}}{l_{22} l_{33}-l_{32} l_{23}}\right], \\
& B_{2}=B_{6}=-\frac{P_{0} l_{23} c_{4}}{q_{2}\left(l_{22} l_{33}-l_{32} l_{23}\right)}, \\
& B_{3}=B_{7}=\frac{P_{0} l_{22} c_{4}}{q_{3}\left(l_{22} l_{33}-l_{32} l_{23}\right)}, \\
& B_{4}=B_{8}=-\frac{P_{0} c_{4}\left[a_{1}\left(l_{12} l_{23}-l_{13} l_{22}\right)-a_{2} l_{11} l_{23}+a_{3} l_{22} l_{11}\right]}{a_{4} q_{4}\left(l_{22} l_{33}-l_{32} l_{23}\right)} .
\end{aligned}
$$

Using these values of $B_{i}{ }^{\prime} s$ in Eqs (3.48)-(3.57), we obtain transformed components of displacement, microrotation, temperature distribution, tangential and normal stress, induced electric field and magnetic field, where

$$
\begin{aligned}
& l_{11}=a_{2} c_{4}-c_{1} a_{4}, \quad l_{12}=a_{2} c_{4}-a_{4} c_{2}, \quad l_{13}=a_{3} c_{4}-a_{4} c_{3}, \\
& l_{22}=\left(d_{2} c_{4}-c_{2} d_{4}\right)-\frac{\left(d_{1} c_{4}-c_{1} d_{4}\right)}{\left(a_{1} c_{4}-c_{1} a_{4}\right)}\left(a_{2} c_{4}-c_{2} a_{4}\right), \\
& l_{23}=\left(d_{3} c_{4}-c_{3} d_{4}\right)-\frac{\left(d_{1} c_{4}-c_{1} d_{4}\right)}{\left(a_{1} c_{4}-c_{1} a_{4}\right)}\left(a_{3} c_{4}-c_{3} a_{4}\right), \\
& l_{32}=\left(b_{2} c_{4}-c_{2} b_{4}\right)-\frac{\left(b_{1} c_{4}-c_{1} b_{4}\right)}{\left(a_{1} c_{4}-c_{1} a_{4}\right)}\left(a_{2} c_{4}-c_{2} a_{4}\right), \\
& l_{33}=\left(b_{3} c_{4}-c_{3} b_{4}\right)-\frac{\left(b_{1} c_{4}-c_{1} b_{4}\right)}{\left(a_{1} c_{4}-c_{1} a_{4}\right)}\left(a_{3} c_{4}-c_{3} a_{4}\right) .
\end{aligned}
$$




\section{Inversion of the transforms}

The transformed components of displacement, microrotation, temperature distribution, tangential and normal stress, couple stress, induced electric field and magnetic field are dependent on $x_{3}, s$ and $\xi$. To obtain them in the physical domain in the form of $f\left(x_{1}, x_{3}, t\right)$, we invert integral transforms by using the inversion technique as used by Singh et al. [18].

\section{Numerical result and discussion}

Following Eringen [19], we take the following values of relevant parameters for the case of magnesium crystal as

$$
\begin{aligned}
& \lambda=9.4 \times 10^{10} \mathrm{~N} / \mathrm{m}^{2}, \quad \mu=4 \times 10^{10} \mathrm{~N} / \mathrm{m}^{2}, \quad K=1 \times 10^{10} \mathrm{~N} / \mathrm{m}^{2}, \quad \rho=1.74 \times 10^{3} \mathrm{~kg} / \mathrm{m}^{3}, \\
& x_{3}=1, \quad j=0.2 \times 10^{-19} \mathrm{~m}^{2}, \quad K^{*}=1.1753 \times 10^{-19} \mathrm{~m}^{2}, \quad \omega^{*}=0.0787 \times 10^{-1} \mathrm{~N} \mathrm{sec} / \mathrm{m}^{2}, \\
& \tau_{0}=6.131 \times 10^{-13} \mathrm{~S}, \quad \tau_{1}=8.765 \times 10^{-13} \mathrm{~s}, \quad \varepsilon=0.073, \quad T_{0}=296 \mathrm{~K}, \\
& \alpha_{0}=0.779 \times 10^{-9} \mathrm{~N}, \quad \in_{0}=\frac{1}{36 \pi} \times 10^{-9} \mathrm{Fm}^{-1}, \quad \mu_{0}=4 \pi \times 10^{-7} \mathrm{Hm}^{-1}, \quad \Omega=1 .
\end{aligned}
$$

The computations are carried out for the non-dimensional time $t=1 / 2$ and range $0 \leq x_{1} \leq 9$. The distribution of non-dimensional normal displacement $u_{3}$, non-dimensional normal stress $\sigma_{33}$, nondimensional tangential couple stress $m_{32}$ and non-dimensional temperature distribution $T$ with nondimensional distance $x_{1}$ have been shown in Figs 1-4.

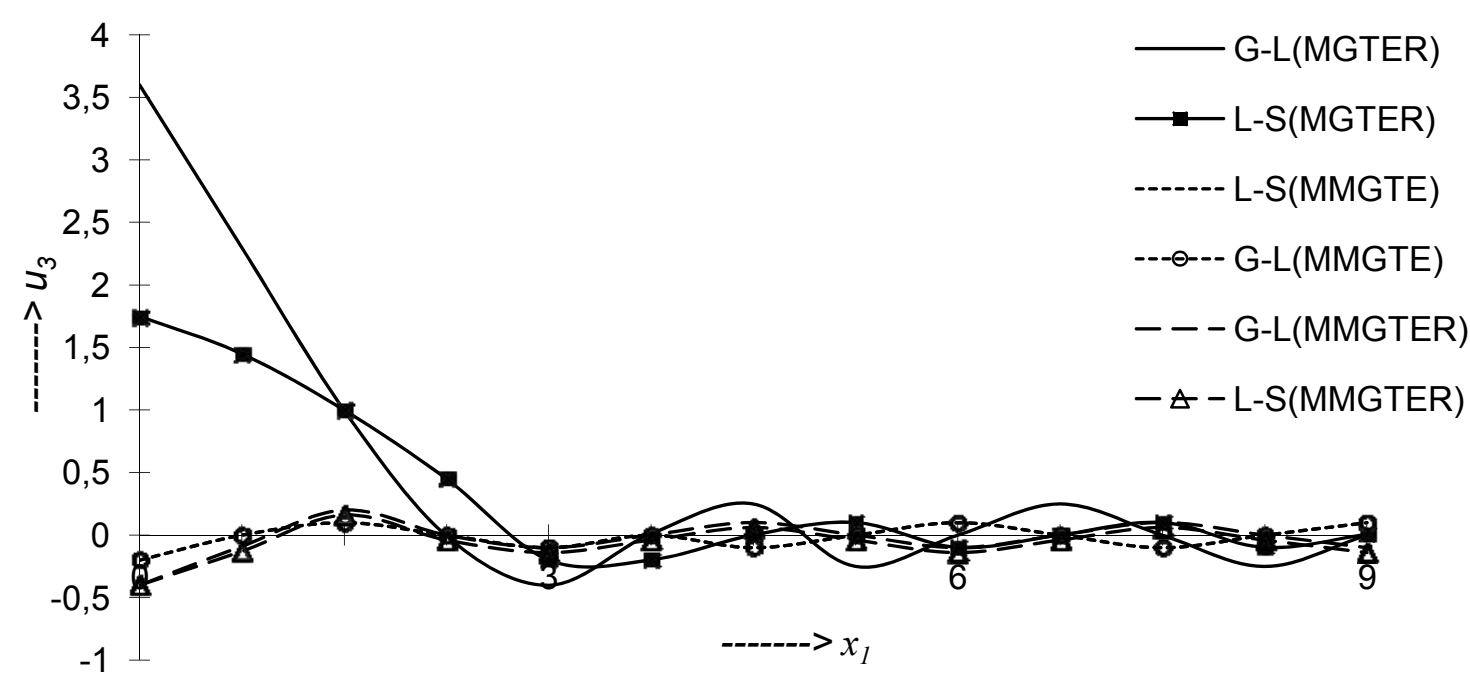

Fig.1. Variation in normal displacement $u_{3}$. 


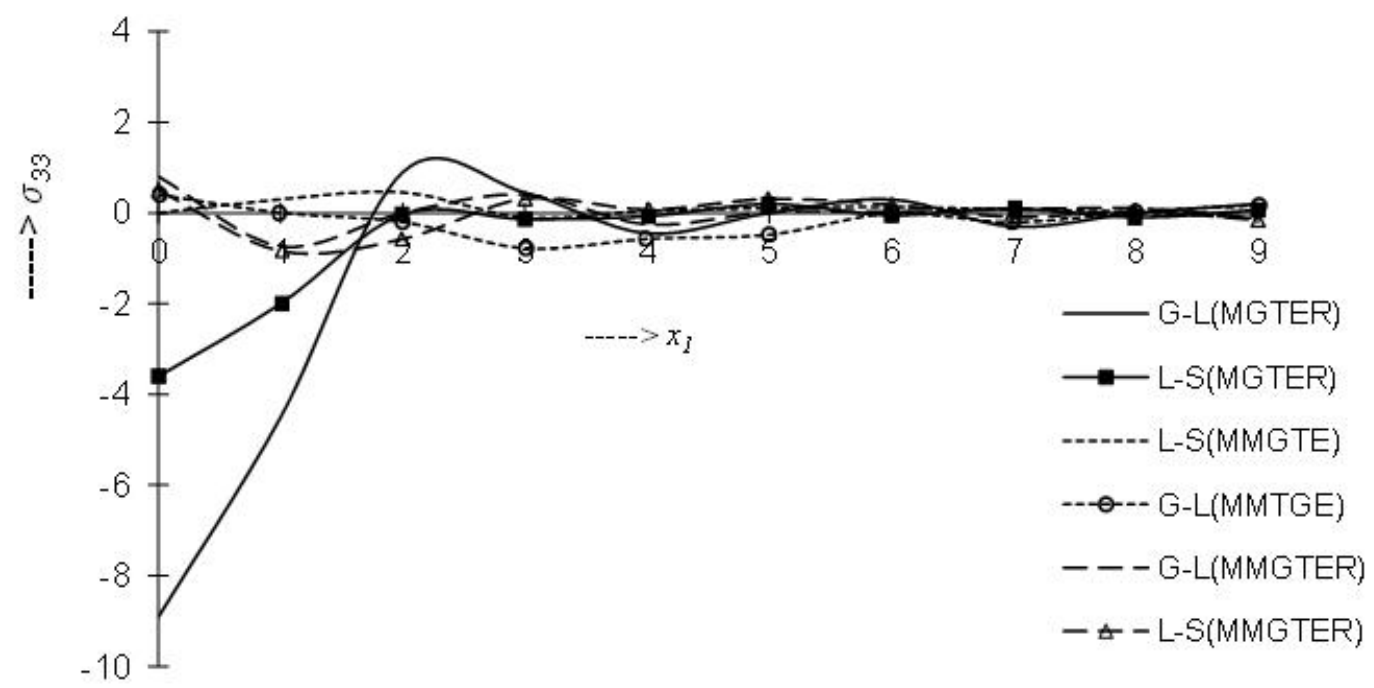

Fig.2. Variation in normal force stress $\sigma_{33}$.

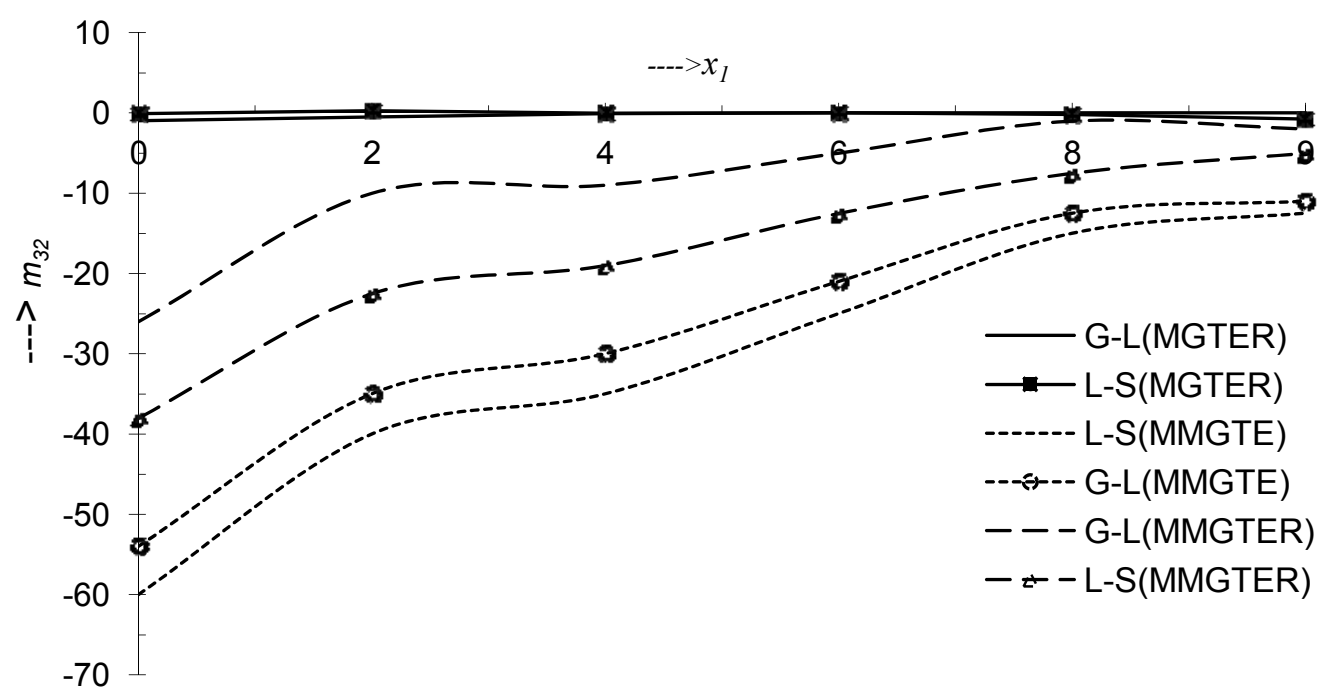

Fig.3. Variation in tangential couple stress $m_{32}$.

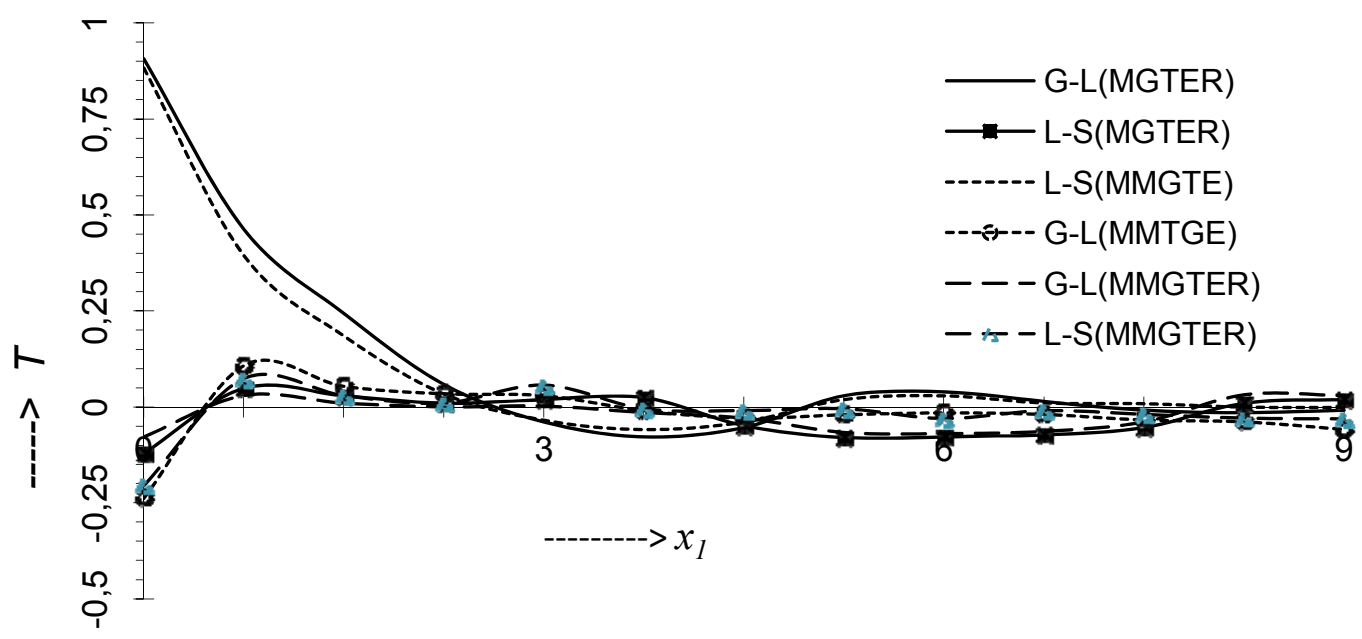

Fig.4. Variation in temperature field $T$. 
The solid line and solid line with solid square represent a micropolar generalized thermoelastic medium, for Green and Lindsay [20] theory as G-L (MGTER) and for Lord and Shulman [21] theory as L-S (MGTER), respectively with the rotation effect. The small dashes line and small dashes line with circles represent a magneto micropolar generalized thermoelastic medium, for L-S theory as L-S (MMGTE) and for G-L theory as G-L (MMGTE), respectively. The large dashes line and large dashes line with triangles represent magneto micropolar generalized thermoelastic medium with the rotation effect, for G-L theory as G-L (MMGTER) and for L-S theory as L-S (MMGTER), respectively. The variations in normal displacement $u_{3}$, normal stress $\sigma_{33}$, tangential couple stress $m_{32}$ and temperature distribution $T$ with distance $x_{1}$ have been shown for mechanical force in Figs 1-4.

It is clear from Fig. 1 that near the source $u_{3}$ has higher values for G-L (MGTER) and L-S (MGTER) theories as compared to its values for all other theories. Also, as $x_{1}$ increases, the electromagnetic and rotation effect tend to diminish. Figure 2 again shows that electromagnetism and the rotation effect have much less impact in the range $3 \leq x_{1} \leq 9$ for normal stress $\sigma_{33}$. Figure 3 shows that tangential couple stress keeps on increasing as we move away from the point of application of the source for all theories. Finally, Fig.4 shows that variation in the temperature distribution $T$ with the rotation effect, near the source has higher values and then keeps on decreasing with $x_{1}$ whereas without the rotation effect it has lower values near the source and then keeps on increasing with $x_{1}$.

\section{Conclusion}

From the above discussion it is evident that normal displacement, normal stress, tangential couple stress and temperature distribution $T$ are affected significantly by the application of rotation and magnetic field. Significant difference can be obtained in the temperature distribution by including the rotation effect. Also, when the case of rotation effect is considered, normal stress shows opposite behaviour for L-S and G-L theories.

\section{Nomenclature}

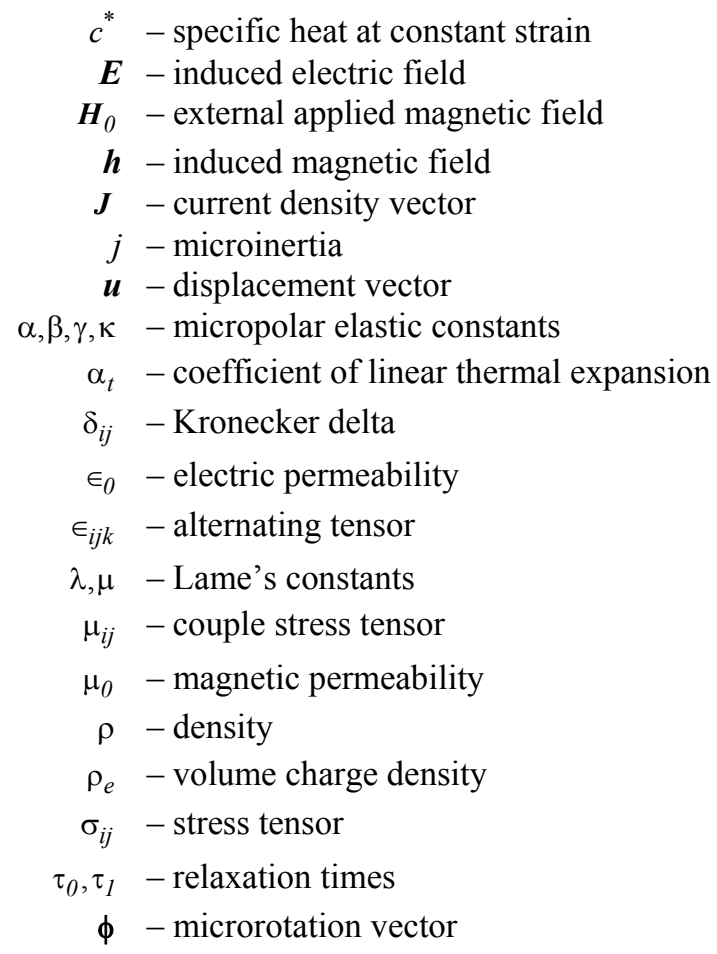




\section{References}

[1] Baksi A., Bera R. and Debnath L. (2005): A study of magneto-thermo elastic problems with thermal relaxation and heat sources in a three dimensional infinite rotating elastic medium. - Int J. Eng. Sci., vol.43, pp.1419-1434.

[2] Banos A. (1956): Normal modes characterizing magneto-elastic plane waves. - Phys. Rev., vol.104, pp.300-305.

[3] Chadwick A. (1957): Elastic wave propagation in magnetic field. IX Cong. - Int. Mech. Appl., vol.7, pp.143-158.

[4] Eringen A. (1966): Linear theory of micropolar elasticity. - J. Math. Mech., vol.15, pp.909-923.

[5] Eringer A. (1984): Plane waves in non local micropolar elasticity. - Int J. Eng. Sci., vol.22, pp.1113-1121.

[6] Ezzat M. and Awad E. (2009): Micropolar generalized magneto-thermoelasticity with modified Ohm's and Fourier's laws. - J. Math. Appl., vol.353, pp.99-113.

[7] Ezzat M. and Bary A. (2009): State space approach of two-temperature magneto-thermoelasticity with thermal relaxation in a medium of perfect conductivity. - Int J. Eng. Sci.,vol. 47, pp.618-630.

[8] Ezzat M. and Youssef H. (2005): Generalized magneto-thermo-elasticity in perfectly conducting medium. - Int. J. Solids Struct., vol.42, pp.6319-6334.

[9] Green A. and Lindsay K. (1972): Thermoelasticities. - J. Elast., vol.2, pp.1-7.

[10] He T. and Cao L. (2009): A problem of generalized magneto-thermoelastic thin slim strip subjected to a moving heat source. - Math. Comput. Model., vol.49, pp.1710-1720.

[11] Kaliski S. (1968): Thermo-magneto-micro-elasticity. - Bull. Acad. Polon. Sci. Tech., vol.16, No.1, pp.7-12.

[12] Kaliski S. and Nowacki W. (1970): Wave-type equations of thermo-magneto-microelasticity (Thermomagnetomicroelasticity wave equations of heat conduction for thermal and coupled peturbations propagating in isotropic medium at finite velocities). - Bull. Acad. Polon. Sci. Tech, vol.18, No.4, pp.155.

[13] Knopoff L. (1955): The interaction between the elastic motions and the magnetic field in electrical conductors. J. Geophys. Res., vol.60, pp.441-455.

[14] Kumar R. and Rupender (2009): Effect of rotation in magneto-micropolar thermoelastic medium due to mechanical and thermal sources. - Chaos, Solitons and Fractals, vol.41, pp.1619-1633.

[15] Lord H. and Shulman Y. (1967): A generalized dynamical theory of thermo elasticity. - Journal of the Mechanics and Physics of Solids, vol.15, pp.299-309.

[16] Nowacki W. (1971): Two dimensional problem of magnetoelasticity. - Bull. Acad. Polon. Sci. Tech., vol.19, vol.4, pp.307-311.

[17] Paria G. (1962): On magneto-thermo-elastic plane waves. - Proc. Cambridge Philos. Soc., vol.58, pp.527-531.

[18] Purshothama C. (1966): Propagation of small disturbances to magneto-elastics. - Proc. Indian Acad. Sci. A., vol.63, pp.53-64.

[19] Singh R. and Kumar V. (2011): Thermo-mechanical deformation in magneto micropolar thermoelastic medium with modified Fourier and Ohm's law. - Int. J. Appl. Mech. Eng., vol.16, No.1, pp.83-105.

[20] Singh R., Kulwant S. and Kumar V. (2011): Response due to mechanical and thermal sources in micropolar generalized thermoelastic medium. - Int. J. Appl. Mech. Eng., vol.16, No.1, pp.107-128.

[21] Youssef H. (2006): Generalized magneto-thermoelasticity in a conducting medium with variable material properties. - Appl. Math. Computation, vol.173, pp.822-833. 